\title{
Slow light
}

Kirk T. McDonald

Joseph Henry Laboratories, Princeton University, Princeton, NJ 08544

(April 3, 1999)

\section{Problem}

Consider a classical model of matter in which spectral lines are associated with oscillators. In particular, consider a gas with two closely spaced spectral lines, $\omega_{1,2}=\omega_{0} \pm \Delta / 2$, where $\Delta \ll \omega_{0}$. Each line has the same damping constant (and spectral width) $\gamma$, where $\gamma \ll \Delta$.

Classically, one might expect these oscillators to correspond to the "V" configuration of atomic levels sketched in Fig. (1a), in which both higher levels can decay to the ground state by emission of photons of frequencies $\omega_{1}$ and $\omega_{2}$. However, quantum mechanics also permits the " $\Lambda$ " configuration sketched in Fig. (1) $b$ ) in which the highest level can decay to the ground state via emission of a photon of frequency $\omega_{1}$ as well as to the intermediate level via emission at frequency $\omega_{2}$.
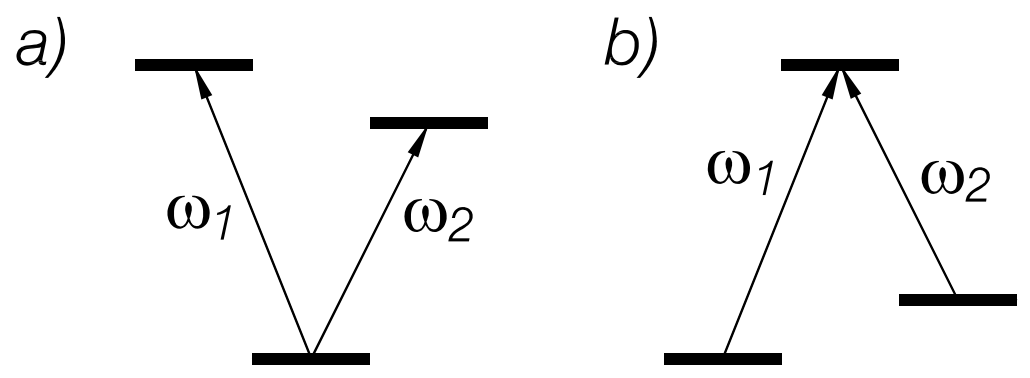

Figure 1: Two possible level diagrams for a three-level atomic system: a) the "V" configuration; b) the " $\Lambda$ " configuration.

The present problem is based on a " $\Lambda$ " configuration in which a laser of frequency $\omega_{2}$ pumps oscillator 2. But since the lower level of this oscillator is not the ground state, the pumping does not result in an inverted population. The physics of this system can be fairly well described by a classical model in which the damping constant of the second oscillator is taken to be negative: $\gamma_{2}=-\gamma$. The strengths of both oscillators are positive.

Deduce an expression for the group velocity of a pulse of light centered on frequency $\omega_{0}$ in this medium. Show also that frequencies very near $\omega_{0}$ propagate without attenuation.

In a recent experiment [1], the group velocity of light was reduced to $38 \mathrm{mph}(17 \mathrm{~m} / \mathrm{s})$ by this technique in a sodium vapor of density $N=8 \times 10^{13}$ atoms $/ \mathrm{cm}^{3}$ using a pair of lines for which $\Delta / 2 \pi \approx 2 \times 10^{7} / \mathrm{s}$.

\section{Solution}

In a medium of index of refraction $n(\omega)$, the dispersion relation can be written

$$
k=\frac{\omega n}{c},
$$


where $k$ is the wave number and $c$ is the speed of light. The group velocity is then given by

$$
v_{g}=\frac{d \omega}{d k}=\frac{1}{d k / d \omega}=\frac{c}{n+\omega \frac{d n}{d \omega}}
$$

We next recall the classical oscillator model for the index of refraction. The index $n$ is the square root of the dielectric constant $\epsilon$, which is in turn related to the atomic polarizability $\alpha$ according to (in Gaussian units)

$$
D=\epsilon E=E+4 \pi P=E(1+4 \pi N \alpha)
$$

where $D$ is the the electric displacement, $E$ is the electric field, $P$ is the polarization density, and $N$ is the number density of atoms. Then,

$$
n=\sqrt{\epsilon} \approx 1+2 \pi N \alpha
$$

for a dilute gas with index near 1 .

The polarizability $\alpha$ is obtained from the dipole moment $p=e x=\alpha E$ induced by electric field $E$. In the case of a single spectral line of frequency $\omega_{0}$, we say that the electron of charge $e$ and mass $m$ is bound to the (fixed) nucleus by a spring of constant $K=m \omega_{0}^{2}$, and the motion is subject to damping $-m \gamma \dot{x}$ where the dot indicates differentiation with respect to time. The equation of motion in the presence of a wave of frequency $\omega$ is

$$
\ddot{x}+\gamma \dot{x}+\omega_{0}^{2} x=\frac{e E}{m}=\frac{e E_{0}}{m} e^{i \omega t} .
$$

Hence,

$$
x=\frac{e E}{m} \frac{1}{\omega_{0}^{2}-\omega^{2}-i \gamma \omega}=\frac{e E}{m} \frac{\omega_{0}^{2}-\omega^{2}+i \gamma \omega}{\left(\omega_{0}^{2}-\omega^{2}\right)^{2}+\gamma^{2} \omega^{2}},
$$

and so the polarizability is

$$
\alpha=\frac{e^{2}}{m} \frac{\omega_{0}^{2}-\omega^{2}+i \gamma \omega}{\left(\omega_{0}^{2}-\omega^{2}\right)^{2}+\gamma^{2} \omega^{2}} .
$$

In the present problem, we have two spectral lines, $\omega_{1,2}=\omega_{0} \pm \Delta / 2$, both of unit oscillator strength, but line 2 is pumped so that $\gamma_{2}=-\gamma_{1}=-\gamma$. In this case, the polarizability is given by

$$
\begin{aligned}
\alpha & =\frac{e^{2}}{m} \frac{\left(\omega_{0}-\Delta / 2\right)^{2}-\omega^{2}+i \gamma \omega}{\left(\left(\omega_{0}-\Delta / 2\right)^{2}-\omega^{2}\right)^{2}+\gamma^{2} \omega^{2}}+\frac{e^{2}}{m} \frac{\left(\omega_{0}+\Delta / 2\right)^{2}-\omega^{2}-i \gamma \omega}{\left(\left(\omega_{0}+\Delta / 2\right)^{2}-\omega^{2}\right)^{2}+\gamma^{2} \omega^{2}} \\
& \approx \frac{e^{2}}{m} \frac{\omega_{0}^{2}-\Delta \omega_{0}-\omega^{2}+i \gamma \omega}{\left(\omega_{0}^{2}-\Delta \omega_{0}-\omega^{2}\right)^{2}+\gamma^{2} \omega^{2}}+\frac{e^{2}}{m} \frac{\omega_{0}^{2}+\Delta \omega_{0}-\omega^{2}-i \gamma \omega}{\left(\omega_{0}^{2}+\Delta \omega_{0}-\omega^{2}\right)^{2}+\gamma^{2} \omega^{2}},
\end{aligned}
$$

where the approximation is obtained by the neglect of terms in $\Delta^{2}$ compared to those in $\Delta \omega_{0}$. The index of refraction (国) corresponding to polarizability (8) is shown in Fig. 2.

We next consider the issue of attenuation of a pulse of frequency $\omega$. Since $k=\omega n / c \approx$ $\omega(1+2 \pi N \alpha) / c$, the spatial dependence $e^{i k z}$ of a pulse propagating in the $z$ direction includes attenuation if the imaginary part of the index $n$ is positive. However, the pumping described by $\gamma_{2}=-\gamma_{1}$ leads to $\operatorname{Im}\left[\alpha\left(\omega_{0}\right)\right]=0$. Hence, there is no attenuation of a probe pulse at 


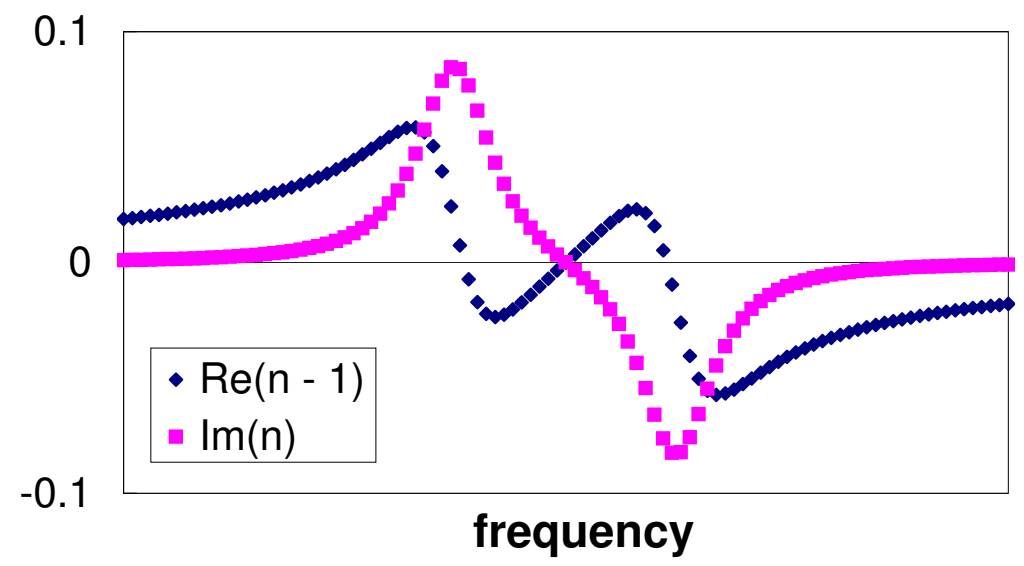

Figure 2: The real and imaginary parts of the index of refraction corresponding to the polarizability (4) in a medium with one of a pair of spectral lines pumped so as to produce a large rate of change of the index with nearby frequency.

frequency $\omega_{0}$. This phenomenon has been called electromagnetically induced transparency [2].

Since $\alpha\left(\omega_{0}\right)=0$, we have $n\left(\omega_{0}\right)=1$, and the phase velocity at $\omega_{0}$ is exactly $c$.

To obtain the group velocity at frequency $\omega_{0}$, we need the derivative

$$
\left.\frac{d R e(n)}{d \omega}\right|_{\omega_{0}}=\left.2 \pi N \frac{d R e(\alpha)}{d \omega}\right|_{\omega_{0}}=\frac{4 \pi N e^{2}\left(\Delta^{2}-\gamma^{2}\right)}{m \omega_{0}\left(\Delta^{2}+\gamma^{2}\right)^{2}} \approx \frac{4 \pi N e^{2}}{m \omega_{0} \Delta^{2}}
$$

where the approximation holds when $\gamma \ll \Delta$ as is the case here. For large density $N$, the group velocity (2) is given by

$$
v_{g} \approx \frac{\Delta^{2}}{4 \pi N r_{0} c}
$$

where $r_{0}=e^{2} / m c^{2} \approx 3 \times 10^{-13} \mathrm{~cm}$ is the classical electron radius. The group velocity is lower in a denser medium.

In the experiment of Hau et al., the medium was sodium vapor, cooled to less than 100 $n K$ to increase the density. An additional increase in density by a factor of 16 was obtained when the vapor formed a Bose condensate. Plugging in the experimental parameters, $N=$ $8 \times 10^{13} / \mathrm{cm}^{3}$ and $\Delta \approx 1.2 \times 10^{8} / \mathrm{s}$, we find $v_{g} \approx 1700 \mathrm{~cm} / \mathrm{s}$ as observed in the lab.

\section{References}

[1] L.V. Hau et al., Light speed reduction to 17 metres per second in an ultracold atomic gas, Nature 397, 594-598 (1999).

[2] S.E. Harris, Eletromagnetically Induced Transparency, Physics Today 50(7), 36-42 (1997). 